\title{
Le processus de décision dans les systèmes
} éducatifs

Introduction

Martine Safra et Odile Luginbühl

\section{OpenEdition}

\section{Journals}

Édition électronique

URL : https://journals.openedition.org/ries/1803

DOI : $10.4000 /$ ries. 1803

ISSN : 2261-4265

\section{Éditeur}

France Education international

\section{Édition imprimée}

Date de publication : 1 avril 2003

Pagination : 25-27

ISSN : $1254-4590$

\section{Référence électronique}

Martine Safra et Odile Luginbühl, «Le processus de décision dans les systèmes éducatifs », Revue internationale d'éducation de Sèvres [En ligne], 32 | avril 2003, mis en ligne le 24 novembre 2011 consulté le 06 juillet 2021. URL : http://journals.openedition.org/ries/1803 ; DOI : https://doi.org/ $10.4000 /$ ries. 1803 


\section{Le processus de décision dans les systèmes éducatifs}

\section{Introduction}

\section{Martine Safra, Odile Luginbühl}

Comment la politique éducative se construit-elle? Comme toujours dans les secteurs où se croisent et peuvent s'opposer les intérêts de la sphère publique (enjeux sociétaux) et de la sphère privée (trajectoires individuelles), la question suscite dans les pays démocratiques une forte demande d'information et, de plus en plus fréquemment, une volonté de participation.

Mais la complexité des facteurs qui interviennent dans l'évolution des systèmes éducatifs est telle que le sens et la justification des décisions prises, c'est-à-dire leur cohérence et leur finalité, manquent souvent de lisibilité aux yeux de l'opinion publique - d'où les sentiments de défiance, voire d'incompréhension, qui peuvent s'exprimer lors de leur mise en place. Les réformes en matière d'éducation peuvent en effet s'effectuer sous diverses influences, dont le poids respectif et l'articulation sont fluctuants selon l'histoire et le contexte de chaque pays: mutations de la société, liées à des changements politiques et/ou à l'évolution des mentalités et des pratiques sociales, renouvellement des connaissances et des théories scientifiques, transformations économiques et technologiques sont autant de phénomènes déclencheurs de nouveaux choix éducatifs.

Ainsi, dans ce dossier, on trouvera l'exemple de l'Espagne et de la Hongrie, où les ruptures politiques avec les régimes antérieurs, le franquisme dans un cas, le communisme dans l'autre, ont à l'évidence entraîné des bouleversements importants marqués par l'émergence de débats, d'hésitations, de contradictions qui reflètent la pluralité des points de vue et constituent une nouvelle donne dans des pays où avait dominé pendant longtemps un parti unique. L'analyse détaillée de décisions prises dans ces deux systèmes illustre tout à la fois la volonté de changement et les difficultés à le mettre en œuvre : Ivan Bajomi montre combien la notion même d'une définition réglementaire des contenus d'enseignement pouvait, dans un contexte d'ouverture et de libéralisation du système éducatif hongrois, prendre une dimension polémique et 
susciter des décisions chaotiques, passant de l'unicité rigide à l'éparpillement local. Les mêmes fluctuations sont observées en Espagne par Juan Carlos Faraco et Diego Sevilla dans un autre domaine clé, celui de la direction de l'établissement scolaire, pris comme exemple de la construction d'une «micropolitique scolaire» soumise à l'alternance démocratique, entre participation de la communauté éducative et gestion professionnalisée. Autre exemple au Japon, décrit par Jean-François Sabouret, où la remise en cause actuelle de certains principes fondateurs de l'éducation dans la loi issue de la Constitution de 1947, tels que l'égalité des chances, est perceptible à travers une série de propositions des commissions en charge de la réforme du système. Ce mouvement semble porté par un regain du sentiment nationaliste dépassant largement le cadre de l'éducation et sous-tendu par les problèmes économiques que connaît le pays.

Cependant, déterminer le substrat idéologique d'une décision, éclairer sa doxa pourrait-on dire, ne suffit pas pour comprendre l'ensemble du mode opératoire de la démarche dans laquelle elle s'inscrit. On ne peut pas davantage isoler l'acte lui-même des phénomènes qui l'ont suscité que des conditions de sa mise en place. La construction d'une politique éducative relève d'un processus d'ensemble, en amont et en aval de la prise de décision, dans un jeu continuel de prise en compte des contraintes multiples et de négociation avec les différents acteurs: cheminement qu'explore Iñes Aguerrondo dans sa présentation de la rénovation du système éducatif argentin, particulièrement éclairante dans la mesure où ce pays, caractérisé par une grande hétérogénéité économique et culturelle des États qui le composent, s'efforce de mettre en place un système fortement décentralisé tout en préservant, dans un souci d'équité, un pilotage par le ministère national. Cette complexité du processus est également traitée par Georges Solaux qui propose, à partir d'exemples français, une approche plus théorique en livrant des outils d'analyse dont les conclusions soulignent le fonctionnement beaucoup plus pragmatique - sur le modèle de la méthode «essais erreurs»- que rationnel et scientifique des prises de décision.

Que nous enseignent ces différents éclairages? On peut, au-delà des spécificités géographiques et historiques, constater certaines convergences dans les situations examinées. La manifestation la plus largement perceptible consiste, derrière le jeu inévitable des changements d'orientations politiques, dans le dessin subtil du difficile équilibre à trouver entre le centre et la périphérie, cette dernière notion pouvant recouvrir des situations très diverses, allant de la région (ou l'État régional) au niveau local de la microstructure. Mais partout, la tendance à la décentralisation pose, d'une part, la question de la régulation nationale et de l'égalité des chances et, d'autre part, celle du rôle respectif des experts et de la collectivité, problèmes que chacun cherche à résoudre dans une démarche relevant plus souvent du tâtonnement que du projet à long terme. 
"Histoire brûlante», pour reprendre la formule de Faraco et Sevilla, semble bien être, quel que soit le pays concerné, l'expression qui caractérise le mieux l'analyse du processus de décision en éducation. Sa complexité, mise en lumière dans l'ensemble des articles, n'a rien pour surprendre si l'on considère l'importance des enjeux véhiculés par les systèmes éducatifs - identité nationale, transmission culturelle, progrès économiques - et, par ailleurs, le paradoxe de leur fonctionnement inscrit, selon les termes de Solaux, dans « un espace social structuré et hiérarchisé, mais ouvert aux stratégies d'acteurs ». La prise en compte de ces données rend primordiale, même sur la base objective d'évaluations externes en amont, la part de la concertation tout au long du processus. 